\title{
RETRACTION
}

\section{Efficacy comparison between minimally invasive and conventional surgery for lumbar disc herniation in Chinese Han population: a meta-analysis}

\author{
S Ji, Q Shao, Y Wang and J Liu
}

Spinal Cord (2017) 55, 626; doi:10.1038/sc.2015.240; published online 18 December 2015

Retraction to: Spinal Cord (2014) 52, 734-739; doi:10.1038/ sc.2014.98; published online 10 June 2014

The Publisher and Editor retract this article in accordance with the recommendations of the Committee on Publication Ethics (COPE).
After a thorough investigation we have strong reason to believe that the peer review process was compromised.

The original article was published online on 10 June 2014 . 